\title{
El curso de inglés técnico constituido como una comunidad de aprendizaje virtual generador de competencias comunicativas en ingeniería civil
}

\author{
Jairo Gutiérrez de Piñeres-Rocha \\ Ingeniería Civil, Fundación Universitaria del Área Andina, Valledupar, Colombia.jgutierrez52@unad.edu.co
}

\begin{abstract}
Resumen - Esta investigación explora cómo el curso de Inglés Técnico en Ingeniería Civil (ITIC) de la Fundación Universitaria del Área Andina (FUAA) se constituye en un modelo de Comunidad de Aprendizaje Virtual (CAV), desde donde se generan unas competencias comunicativas [1]. Se intenta indagar cómo se aprende la lengua Inglesa a través del ITIC y qué destrezas se adquieren cuando se interactúa en una CAV. Por tal razón es relevante identificar los componentes de las competencias comunicativas para potenciar las actividades de la lengua Inglesa como la comprensión, la expresión y la interacción de los estudiantes dentro de una CAV. En consecuencia esta investigación se constituye en una primera fase exploratoria enfocada en primera instancia hacia los estudiantes matriculados en el ITIC como un rasgo distintivo de validez interna.
\end{abstract}

Palabras Clave- Programa Ingeniería Civil, Inglés Técnico, Competencias Comunicativas, Comunidad de Aprendizaje Virtual.

Recibido: 24 de Abril de 2017. Revisado: 29 de Junio de 2017 Aceptado: 18 de julio de 2017

\section{Bilingualism national program created as a virtual learning community generating commucation skills in civil engineering}

Abstract - This research explores how the Technical English of Civil Engineering (TECE) of Fundación Universitaria del Área Andina (FUAA) is a model of Virtual Learning Community (CAV), according to the model proposed by Pratt y Palloff (2007), where it generated some communication skills. It seeks to identify how people learn the English language through the TECE and what skills are acquired when you interact in a CAV. For this reason it is important to identify the components of communication skills to enhance the activities of the English language and comprehension, expression and interaction of students within a CAV. Consequently, this research constitutes an exploratory first phase focused primarily on students enrolled in the TECE as a distinctive feature of internal validity.

Keywords- Civil Engineering Program, Technical English, Communicative Competences, Virtual Learning Community.

\section{Introducción}

El curso de inglés técnico en Ingeniería Civil (ITIC) es un núcleo formativo en inglés como lengua extranjera ofertado por la Fundación Universitaria del Área Andina (FUAA). El ITIC se diseñó e implementó en el 2016 para promover el uso del inglés técnico con fines formativos basados en el modelo de aprendizaje autónomo entre toda la población estudiantil a través de sus cuatro (4) niveles. En consecuencia, el ITIC se oferta a través de las dos mediaciones pedagógicas contempladas por la ITIC, por el Sistema Tradicional y por Campus Virtual; pero a partir de 2016 se comenzó a ofertar sólo por Campus Virtual y muy excepcionalmente por la primera mediación. Por tal razón, podríamos ubicar al ITIC en la categoría de AvDtDe (alta virtualidad, diferente tiempo, diferente espacio), en el Modelo Tridimensional [2] y soportada en las categorías de transición [3], a partir de la decisión de Vicerrectoría Académica y de la Coordinación del programa de FUAA.

$\mathrm{Si}$ el ITIC se posiciona en la AvDtDe, debemos mencionar el aprovechamiento de la funcionalidad y la eficiencia de la tecnología y los recursos del Internet con fines formativos maximizando la versatilidad del ambiente virtual de aprendizaje, espacio definido como el entorno en donde los estudiantes interactúan con sus tutores, demás estudiantes y con los contenidos, constituyéndose así en una Comunidad de Aprendizaje Virtual (CAV) en donde el estudiante usa la lengua para comprender, adquirir y desarrollar destrezas productoras y receptoras del Inglés como lengua extranjera.

Los cuatro niveles del ITIC fueron diseñados bajo el enfoque comunicacional y la doctrina naturalista de adquisición del lenguaje según [4]. Estos enfoques requieren de la interacción y la realimentación permanente de los actores académicos en una CAV para la práctica y desarrollo de las competencias orales [4].

En consecuencia, el objetivo de la presente investigación es determinar cómo el ITIC mediante la estructura formativa de una $\mathrm{CAV}$, puede generar competencias comunicativas a los estudiantes definidos como agentes sociales activos dentro del ITIC caracterizado como un ambiente virtual de aprendizaje ofertados por la FUAA.

Por consiguiente, las personas miembros de una CAV utilizan las competencias disponibles en los multicontextos con fines comunicativos y sociales que conlleven a una interacción formativa. Pero a la vez, estas competencias están restringidas y condicionadas

Como citar este artículo: Piñeres-Rocha, J.G., El curso de inglés técnico constituido como una comunidad de aprendizaje virtual generador de competencias comunicativas en Ingeniería Civil. Educación en Ingeniería, 12(24), pp. 101-106, Julio, 2017. 
por el mismo multicontexto para realizar actividades de bilingüismo, en este caso el inglés, que conllevan a procesos comunicativos productivos y receptivos con temas en ámbitos específicos poniendo en juego las estrategias pedagógicas y didácticas más apropiadas para llevar a cabo las tareas que se han de realizar [1]. Por tal razón, el control y dominio que de estas acciones tengan las personas dentro de la CAV producirá el refuerzo o la modificación de sus competencias [5].

\section{Marco teórico}

Inicialmente se declararía el concepto de competencia por algunos expertos de la Sociedad del Conocimiento Global y luego identificar el ITIC como una CAV generadora de competencias comunicativas; pero no sin antes describir y analizar el acto lingüístico como antecesor de las primeras.

Una competencia es la suma de conocimientos, destrezas y características individuales que permiten a una persona realizar acciones [5]; y una competencia comunicativa es la que posibilita a una persona actuar utilizando específicamente medios lingüísticos, y a la vez, implican actividades de lengua que suponen el ejercicio de la competencia lingüística comunicativa dentro de un ámbito específico a la hora de procesar (en forma de comprensión o de expresión) uno o más textos con el fin de realizar una tarea [5].

Por tal razón, este ejercicio lingüístico conlleva a la definición de competencia lingüística como la capacidad que tiene todo ser humano de manera innata de poder hablar y crear mensajes que nunca antes había oído [6]. Esta competencia se centra en las operaciones gramaticales que tiene interiorizado el individuo y se activan según se desarrolle su capacidad coloquial. Es decir, el lenguaje nace desde dentro del individuo y no desde lo social [7]. El maestro lo que tiene que hacer es desarrollar esta competencia lingüística en el alumno haciéndole que hable y enseñándole vocabulario y no sólo gramática. La competencia lingüística se hace realidad a través de reglas generativas que se relacionan con la gramática que es saber organizarse y estructurarse.

Más que una yuxtaposición al concepto de competencia lingüística, aparecería como complemento la noción de competencia comunicativa referida al uso del lenguaje en actos de comunicación particulares, concretos y social e históricamente situados, introduciendo una visión más pragmática del lenguaje, en la que los aspectos socio-culturales resultan determinantes en los actos comunicativos [8].

Continuando con la competencia lingüística como elemento constitutivo de la competencia comunicativa, definida como la parte integral de la inteligencia que parece compartida de manera más universal y democrática en toda la especie humana [9]. Así mismo, se considera a la fonología y la sintaxis como las estructuras de tal tipo de inteligencia, mientras que la semántica y la pragmática se relacionan más con otros tipos de inteligencias [9]. En esta forma se podría definir este ejercicio lingüístico como una competencia en la cual se permitiría procesar información de un sistema de símbolos para reconocer la validez fonológica, sintáctica o semántica en un acto de significación de esa lengua.

Fundamentados en las anteriores definiciones y conceptos, podríamos comenzar a proponer el ITIC como una CAV en donde se aplica e implementa todo el soporte teórico de los expertos en lingüística para identificar la generación de competencias comunicativas que le permita a los estudiantes procesar toda la información de un sistema de símbolos para reconocer la validez fonológica, sintáctica o semántica en un acto de significación de esa lengua en un acto específico.

De igual forma y en concordancia con la funcionalidad formativa del ITIC, es relevante describir que el ITIC se presenta y se ajusta a la estructura de una CAV por todos los elementos que intervienen y se entrelazan conformando unos subsistemas críticos y transformadores como categorías taxonómicas que dentro de los conceptos de las redes globales de aprendizaje se manifiestan la interacción y la interactividad entre todos sus actores académicos para la generación reflexiva y solidaria de competencias comunicativas en la Ingeniería Civil.

Por consiguiente, se explicará al detalle por qué el ITIC se constituye como una CAV, y cómo a través de su orden estructural y morfológico fomenta competencias comunicativas en lo lingüístico, en lo sociolingüístico y en lo pragmático como componentes esenciales en la Ingeniería Civil [5]. Por tal razón, se aplicó una encuesta cerrada a una muestra de 30 estudiantes (quienes respondieron la encuesta) para recolectar la información pertinente que permitiera ponderar los componentes de la competencia comunicativa.

\section{Metodología}

Se decidió en primera instancia caracterizar al ITIC como una estructura de CAV y sus tres elementos constitutivos [1]: personas, proceso y propósito, y observar como el ITIC se ajusta de una manera interactiva y versátil al mismo con el objetivo de generar competencias comunicativas en inglés bajo el contexto de la Ingeniería Civil.

Se hizo de esta forma, porque el ITIC aparte de tener todos los elementos implícitos pero aún no claramente delimitados del modelo propuesto, posee la misma funcionalidad formativa en cuanto a los diseños de logros de aprendizaje para garantizar la generación de competencias comunicativas de una forma interactiva, colaborativa y asertiva.

La presente investigación es de tipo exploratorio, en donde se aplican dos procedimientos instrumentales para caracterizar las competencias comunicativas generadas por el ITIC dentro del modelo de CAV: El primero se refiere a los componentes contemplados por el [5], como son los lingüísticos, sociolingüísticos y pragmáticos, los cuales a la vez, se constituyen en los indicadores para caracterizar la competencia comunicativa dentro del ITIC con una semántica y semiología aplicada a la Ingeniería Civil.

En consecuencia, la encuesta aplicada permite observar la generación de las competencias comunicativas en la Ingeniería Civil a través de estos componentes porque se estaría indagando por lo siguiente:

- Las competencias Lingüísticas incluyen los conocimientos y las destrezas léxicas, fonológicas y sintácticas, y otras dimensiones de la lengua como sistema aplicadas a la disciplina, independientemente del valor sociolingüístico de sus variantes y de las funciones de sus realizaciones.

- Las competencias Sociolingüísticas se refieren a las condiciones socioculturales del uso de la lengua en el programa.

- Las competencias Pragmáticas tienen que ver con el uso funcional de los recursos lingüísticos (producción de funciones de lengua, de actos de habla) sobre la base de guiones o 
escenarios de intercambios comunicativos referidos a la Ingeniería Civil.

En el segundo procedimiento se analizan las cuatro destrezas en el ITIC mediante otra encuesta con preguntas orientadoras con el fin de establecer los efectos de interacción oral y de interacción escrita en los estudiantes de aula en estudio y su efecto en la generación de competencias comunicativas en la Ingeniería Civil.

Por tal razón, en ambos procedimientos el objetivo principal era definir si los componentes y las cuatro destrezas seleccionadas como actividades de la lengua en cuanto a la comprensión y a la expresión como procesos primarios para lograr la interacción comunicativa efectiva dentro del ITIC se podían constituir en fundamento válido para determinar la generación de competencias comunicativas.

Por consiguiente, para ambos procedimientos se procedió a elaborar y probar el instrumento de recolección de información a través de dos encuestas cerradas, enviada a los correos personales de los estudiantes, utilizando una escala de Lickert (1932) de cinco puntos como:

- TA Totalmente de acuerdo

- A De acuerdo

- I Indeciso

- D En desacuerdo

- TD Totalmente en desacuerdo

Asignando el puntaje más alto de 5 a TA, y así sucesivamente hasta 1 a TD.

Para contextualizarse en el ITIC de manera concreta, se recurrió a los protocolos de los niveles de los cursos de inglés como Lengua Extranjera para observar cómo se asumen las competencias en el enfoque curricular del ITIC y correlacionarlas con las actividades evaluativas del curso en el ámbito de la Ingeniería Civil.

El curso ITIC tiene 4 unidades o módulos en su contenido en línea con las siguientes categorías temáticas: Objetivos (funciones de la lengua), vocabulario, lecturas, gramática, pronunciación, escritura.

Adicionalmente, las aulas del ITIC cuentan con recursos sincrónicos, asincrónicos y Objetos Virtuales de Aprendizaje (OVA) y Objetos Virtuales de Información (OVI) que facilitan la interacción y la interactividad dentro de la CAV:

Foros: recursos asincrónicos.

Quices y Examen: recursos sincrónicos.

Fonética y Glosario del Curso: OVA y OVI.

De la población total de 67 estudiantes del curso ITIC, se escogieron 30 estudiantes que representa aproximadamente al 45\%, porcentaje al cual se le aplicó la encuesta por medio de los correos personales y el correo interno del mismo curso ITIC.

\subsection{El curso de Inglés Técnico en Ingeniería Civil (ITIC) constituido como una comunidad de aprendizaje virtual (CAV)}

Se ha descrito a una CAV como un orden o agregado social que utiliza la red como tecnología de distribución de la información a través de un currículo con fines formativos [10], y se complementa con la adición de tres elementos constitutivos: las personas, los propósitos y el proceso en donde se garantice el aprendizaje colaborativo y la práctica reflexiva para que ocurra el aprendizaje transformador [1]. Es en este modelo de CAV en la cual se basará el ITIC para generar competencias comunicativas [1]. Por tal razón, es fundamental caracterizar al ITIC como una CAV para fomentar las competencias comunicativas, las cuales se define como el uso del lenguaje para una acción de comunicación particular, concreta y social e históricamente situada para introducir una visión más pragmática del lenguaje, en la que la interacción y el aprendizaje colaborativo resultan determinantes en los actos comunicativos [8].

El ITIC representa ese agregado social basado en un currículo inmerso en una CAV que fomenta tanto destrezas receptivas (listening, reading) como productivas (speaking, writing), y por consiguiente competencias comunicativas en todos los recursos implementados en el aula. La estructura como una comunidad de aprendizaje virtual (CAV) se podría ajustar dentro de la siguiente tipología:

\subsubsection{Las personas como primer elemento}

Conformado por el director del curso, el tutor de apoyo y los estudiantes. Se tomaron los datos de Inglés Técnico (aula objeto de la investigación) del ITIC de 2016-03, cuyo código de aula es ID0253, podríamos reseñar un director de curso, 2 tutores de apoyo y 67 estudiantes matriculados en el aula. La asignación de los estudiantes es proporcional a la modalidad contractual del tutor de apoyo, y se hace en pequeños grupos colaborativos de máximo 5 (cinco) estudiantes. En consecuencia, la distribución es la siguiente:

TUTOR DE APOYO 1: Tenía 34 estudiantes.

TUTOR DE APOYO 2: Tenía 33 estudiantes.

Se define al elemento personas como indispensable para conformar comunidades y a su vez, se reconocen tres variables críticas inmersas en este elemento: La presencia social, la interacción y la comunicación [1]. Es imprescindible definir a continuación el rol funcional y asistencial de cada persona dentro de la CAV para correlacionar las tres variables críticas que generan competencias comunicativas.

El rol del director es el de planificar, diseñar todas las actividades evaluativas asincrónicas y sincrónicas conforme a un cronograma de trabajo denominado agenda; adicionalmente la organización y la navegabilidad de los recursos dispuestos en cualquier aula del ITIC.

En el mismo sentido, el rol del tutor de apoyo es el de realizar el acompañamiento a los procesos de aprendizaje de los estudiantes mediante la puesta en funcionamiento de estrategias de asesoría a las temáticas de cada nivel del ITIC, orientación sobre los métodos de estudios, seguimiento, evaluación y realimentación de las competencias lingüísticas y comunicativas que el estudiante va adquiriendo acorde con los parámetros establecidos por el ITIC.

El estudiante es el centro de la comunidad de aprendizaje virtual y del proceso formativo que se fomenta en la FUAA, y es quien realiza el aprendizaje autónomo basado en el estudio independiente o trabajo personal para adquirir las competencias lingüísticas y comunicativas a través del ITIC.

En el 2016 se comenzó con la aplicación de unos instrumentos virtuales de recolección de información que sustentan las tres variables críticas de este primer elemento, en donde se visibiliza el fomento de competencias comunicativas producidas por la misión de generar un conocimiento compartido del inglés técnico en la ingeniería civil dentro del aula.

En las aulas del ITIC se cuenta con el recurso asincrónico de dos Foros de Trabajo Colaborativo, uno de actividad evaluativa referente a Writing (destreza productiva) y otro sobre Speaking 
(destreza productiva). Se procede a definir cada recurso y su responsabilidad en la generación de competencias según el instrumento aplicado:

Foro General: Espacio para que interactúen los tutores con sus estudiantes brindándole la orientación en el proceso en general, la solución a las preguntas, inquietudes derivadas del desempeño y la navegabilidad del estudiante dentro del aula. Interacción que se hace de estudiante a estudiante también porque es un ambiente de acceso compartido y masivo.

De los 67, 45 estudiantes usaron este Foro para el fin adecuado, reforzando la presencia social para interactuar con su tutor y demás compañeros de grupo colaborativo. Acción que genera competencias comunicativas porque el estudiante despeja sus dudas, disipa los temores iniciales tanto con su tutor de apoyo y sus pares académicos dentro del contexto.

Foro Colaborativo de Writing: Espacio en donde el grupo colaborativo diseña un producto final evaluado por el tutor de apoyo, el cual es un constructo escrito compartido y solidario de cada miembro del grupo [12]. En primera instancia, los estudiantes hacen sus aportes de manera individual; y luego, se expresa el acuerdo o las sugerencias de ampliación de los conceptos ya emitidos de forma crítico-reflexiva. Posteriormente, se identifican los desacuerdos con argumentos para empezar a diseñar el producto final del grupo colaborativo que se somete a una revisión final antes de presentarlo al tutor de manera definitiva.

Foro Colaborativo de Speaking: Espacio que sigue la misma metodología del foro anterior, pero la diferencia en este caso, es que el producto final contempla un audio grabado en MP3 por los estudiantes del grupo colaborativo. En ese sentido fomentó a los 634 estudiantes participantes, la destreza productiva de comunicarse oralmente, primero entre pares y luego con el tutor de apoyo.

\subsubsection{El propósito como segundo elemento}

En este segundo elemento de una CAV, se definen los lineamientos y reglas de juego de las aulas del ITIC, como también las consideraciones prácticas como por ejemplo los logros de aprendizaje de cada una de las 4 unidades o módulos del ITIC, los tiempos de apertura de las actividades evaluativas y del tiempo de duración de cada una de ellas, etc., como también del tamaño del grupo, en nuestras aulas son un número máximo de 5 estudiantes por grupo colaborativo, y hasta las disposiciones de herramientas metodológicas para crear un sentido de seguridad y privacidad. Elementos básicos para fomentar una adecuada interacción y colaboración dentro de una CAV.

Se caracteriza a través del Protocolo Académico, en donde se establece el lineamiento general del curso, en cuanto a las intencionalidades formativas, las unidades didácticas, el sistema de evaluación, la metodología y las fuentes documentales. Adicionalmente de manera complementaria, la Guía de Actividades, en donde se establece la situación didáctica que delimita la temática de un diseño de aprendizaje.

También se incluyó el chat como herramienta sincrónica y el Foro General como herramienta asincrónica para interactuar con los estudiantes con respecto a las consideraciones prácticas y acordar ciertos criterios como lineamientos.

Estas herramientas fueron caracterizadas en este elemento por el fundamento que demanda el aprendizaje autónomo dentro de la autorregulación adquirida por los estudiantes dentro del ITIC.

\section{Competencias Primer Elemento}

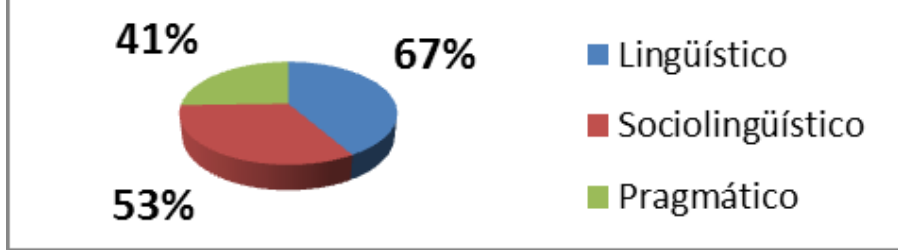

Figura 1. Gráfico de las Competencias adquiridas por componentes en el primer elemento de una CAV.

Fuente: El autor

\section{Competencias Segundo Elemento}

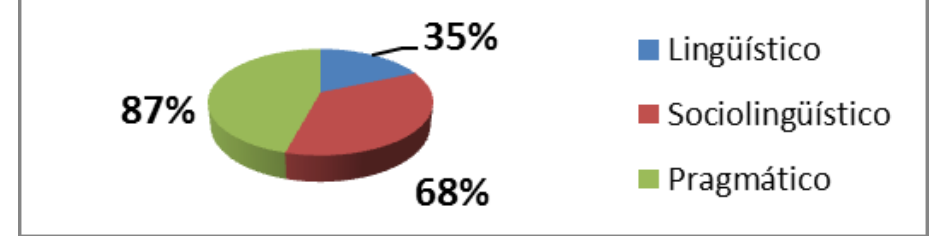

Figura 2. Gráfica de las Competencias adquiridas por componentes en el segundo elemento de una CAV.

Fuente: El Autor.

\subsubsection{El proceso como tercer elemento}

Para que el aprendizaje transformador y el aprendizaje reflexivo el ITIC cuenta con unas actividades evaluativas sincrónicas como el quiz, la lección evaluativa y recursos interactivos en Flash Player y Multimedias en los contenidos en línea que le permiten a los estudiantes adquirir destrezas receptivas como el audio y la lectura de funciones específicas del Inglés; complementados con actividades de vocabulario y gramática.

\subsection{Resultados con respecto al primer elemento de la CAV}

En este foro participaron 45 estudiantes, pero sólo 31 diligenciaron la encuesta. Los porcentajes reportados corresponden solamente a quienes escogieron Totalmente de Acuerdo (TA). En ese orden de ideas, se reporta un $67 \%$ en la adquisición del componente lingüístico, un $53 \%$ en el componente sociolingüístico y un $41 \%$ en el componente pragmático como se muestra en la Fig. 1.

\subsection{Resultados con respecto al segundo elemento de la CAV}

Se reportó un $35 \%$ en la adquisición del componente lingüístico, un 68\% en el componente sociolingüístico y un $87 \%$ en el componente pragmático como se muestra en la Fig. 2. 


\section{Competencias Tercer Elemento}

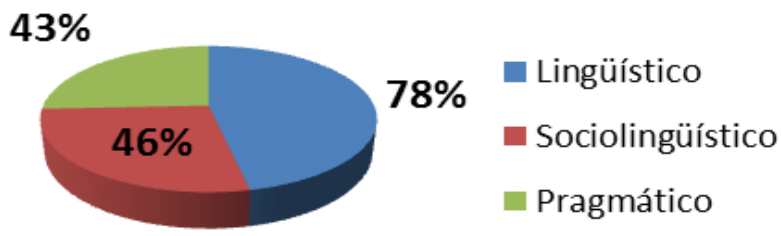

Figura 3. Gráfica de las Competencias adquiridas por componentes en el tercer elemento de una CAV.

Fuente: El Autor.

\subsection{Resultados con respecto al tercer elemento de la CAV}

Se reportó un 78\% en la adquisición del componente lingüístico, un $46 \%$ en el componente sociolingüístico y un $43 \%$ en el componente pragmático en la Fig.3.

\section{Resultados}

En los siguientes puntos se describen los resultados obtenidos por cada una de las interacciones dentro del ITIC.

\subsection{Resultados en cuanto a la interacción oral y a la interacción escrita dentro del ITIC para generar competencias comunicativas}

Adicionalmente a los componentes definidos anteriormente para caracterizar las competencias comunicativas, en este segundo procedimiento se aplicó una segunda encuesta siguiendo el mismo método [11], en donde se incluyeron preguntas orientadoras para identificar la interacción oral y la interacción escrita inmersas dentro de las cuatro destrezas que genera el ITIC. En consecuencia, también se tuvo en cuenta sólo quienes escogieron estar TA en las estadísticas que reflejaron la adquisición de destrezas receptivas y destrezas productivas, las cuales se enuncian a continuación:

\subsubsection{Destrezas receptivas lectoras (DR) según la Fig.4.}

1. Comprende y sigue instrucciones: $87 \%$

2. Identifica ideas principales y secundarias: $55 \%$

3. Hace inferencias lógicas a partir de un texto: $41 \%$

4. Desarrolla el pensamiento crítico y la destreza de leer: $33 \%$

\subsubsection{Destrezas receptivas auditivas (DR) según la Fig.5.}

1. Comprende la información que se entrega en actividades para desarrollar la destreza de escuchar: 54\%

2. Comprende ideas básicas formuladas en inglés hablado: $67 \%$

3. Escucha y responde a las ideas de otros: $49 \%$

\section{DR-LECTORAS}

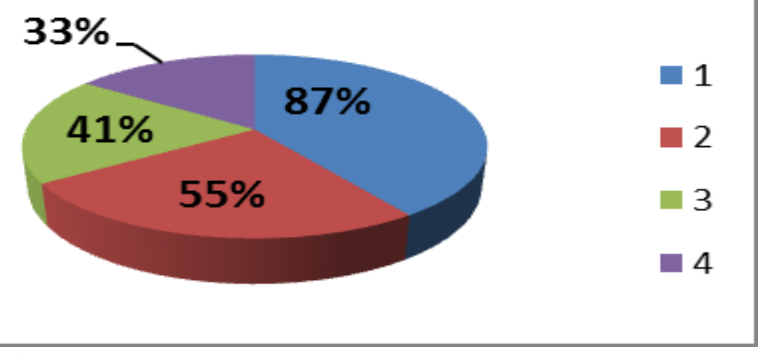

Figura 4. Gráfica de los Porcentaje de las destrezas receptivas lectoras. Fuente: El Autor.

\section{DR-AUDITIVAS}

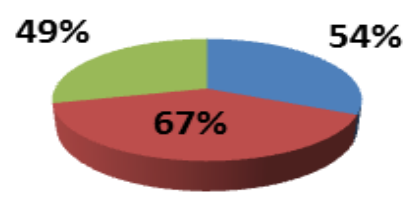

\section{5

Figura 5. Gráfico de los Porcentaje de destrezas receptivas auditivas. Fuente: El autor

\section{DP-ESCRITURA}

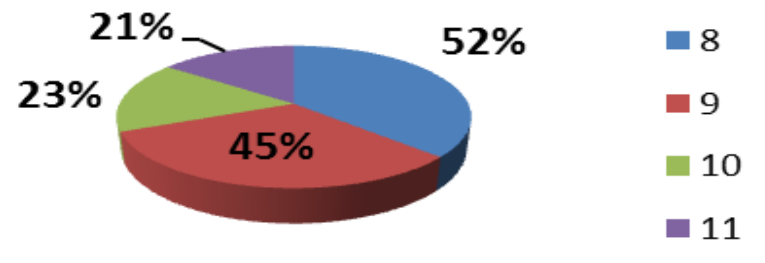

Figura 6. Gráfico de los Porcentaje de destreza productiva en la escritura. Fuente: El autor

\subsubsection{Destreza productiva en la escritura (DP) según la Fig.6.}

1. Toma apuntes a partir de textos escritos: $52 \%$

2. Lee para corregir ideas, organiza un vocabulario en un primer borrador: $45 \%$

3. Utiliza un bosquejo para desarrollar sus ideas: $23 \%$

4. Elabora y redacta composiciones lógicas: $21 \%$

4.1.4. Destreza productiva en el habla (DP) según la Fig.7.

1. Utiliza estructuras gramaticales correctamente: $61 \%$

2. Lleva a cabo presentaciones haciendo un correcto manejo de su pronunciación: $15 \%$

3. Pronuncia correctamente siguiendo los signos de puntuación, con fluidez y buena entonación: 19\% 


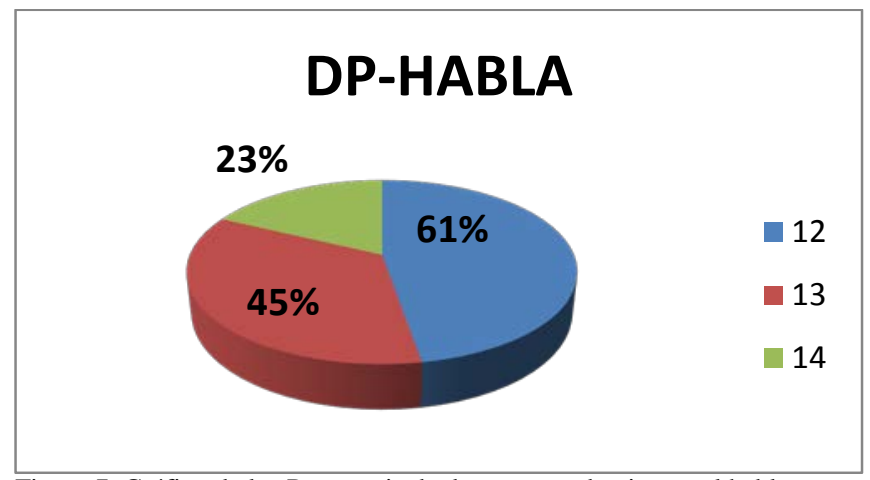

Figura 7. Gráfico de los Porcentaje de destreza productiva en el habla. Fuente: El autor

\section{Conclusiones}

El ITIC genera competencias comunicativas bajo el contexto de comunidad de aprendizaje virtual en el programa de Ingeniería Civil, porque como arrojan las estadísticas, el elemento personas fomenta un aprendizaje colaborativo a través de la interacción y la presencia social, aspectos complementarios en la adquisición de competencias comunicativas.

Por otra parte, dentro del segundo elemento de una CAV, el propósito extrapola unas consideraciones prácticas que son compartidas y aceptadas por los estudiantes mediante la utilización de herramientas sincrónicas y asincrónicas para reforzar el componente sociolingǘstico como un indicador de competencia comunicativa.

Referenciando al tercer elemento de una CAV, proceso, se podría afirmar que bajo el uso de las herramientas sincrónicas y asincrónicas del ITIC se incrementa el aprendizaje transformador y reflexivo porque se crea un ambiente virtual que garantiza las interacciones equitativas y participativas entre el tutor-estudiante y estudiante-estudiante para reforzar la competencia comunicativa como acto pragmático del uso de la lengua. Estas interacciones grupales resultan más significativas que la de la relación aislada entre el tutor y el estudiante, [1].

Por consiguiente, la inclusión de la interacción oral y la interacción escrita en la investigación y su relación con las competencias comunicativas, se evidencia y se potencia (aunque en bajos porcentajes en algunas) a través de las cuatro destrezas del ITIC como un criterio formativo de interacción comunicativasocial.

Adicionalmente, de la investigación se concluye y se propone como tema de discusión que las interacciones orales tienen un grado diferencial de las expresiones y las comprensiones orales en cuanto a la superposición de ambos procesos y la naturaleza de las respuestas en las actividades de Listening y de Speaking; por consiguiente se hace imperativo y necesario que el tutor replantee su rol de orientador apuntándole a la mediación comprensiva y expresiva del estudiante en la retroalimentación sincrónica resaltando la importancia de la fonética y de la respuesta planificada adecuada para fortalecer ambas destrezas en aulas virtuales.

\section{Bibliografía}

[1] Palloff, R. and Pratt, K., Assessment, academic integrity, and community online. Chapter published in Encyclopedia of Distance Learning, Second Edition pp. 108-114. DOI:10.4018/978-1-60566-198-8.ch01

[2] Silvio, J., El liderazgo en la gestión de la calidad de la educación a distancia como innovación. RIED. Revista Iberoamericana de Educación a Distancia, 7(1-2), pp. 17-39, 2004. DOI:10.5944/ried.7.1-2.1073.

[3] Oblinger, D., Global education: Thinking creatively. Higher Education in Europe, 24(2), 251-258, 1999. DOI:10.1080/0379772990240212.

[4] Richards, J., Whole language. In: Approaches and methods in language teaching, 2001, pp.108-114. DOI:10.1017/cbo9780511667305.012.

[5] Common European Framework of Reference (CEFR) on languages, in: Immigration and Membership Politics in Western Europe pp. 243-243. DOI:10.1017/cbo9781107477865.010.

[6] Lightfoot, D.W. and Chomsky, N., Syntactic structures. Berlin, Boston: De Gruyter Mouton, 2002. DOI:10.1515/9783110218329.

[7] De Saussure, F., De Gorog, R. and Baskin, W., Course in general linguistics. The Modern Language Journal, 45(3), pp. 145. 1961 DOI: 10.2307/320587.

[8] Hymes, D., The concept of communicative competence revisited. In: Thirty Years of Linguistic Evolution 1992, 31 P. DOI:10.1075/z.61.08hym.

[9] Gardner, H., First course in mind, brain, and education. Mind, Brain, and Education, 1(2), pp. 61-65, 2007. DOI:10.1111/j.1751228x.2007.00007.x

[10] Cabero-Almenara, J. and Marín-Díaz, V. Campus Virtuales Compartidos (CVC): Análisis de una experiencia. Educación XX1, 14(2), pp. 111-132, 2011 DOI:10.5944/educxx1.14.2.247.

[11] Turner, J., Using likert scales in L2 research. Another researcher comments. Journal TESOL Quarterly, 27(4), pp. 736-739. 1993. DOI:10.2307/3587409.

[12] Gunawardena, Ch., Culture and online distance learning. in Handbook of Distance Education. DOI:10.4324/9780203803738.ch12.

J.A. Gutiérrez de Piñeres-Rocha, recibió el título de Ing. Civil en 1995 de la Universidad Industrial de Santander - UIS, Colombia, el título de MSc. en Online Education en 2010 de la UNAD Florida y doctorando en Ciencias de la Educación en la Universidad de Cuauthemoc de Méjico. Desde el 2005 ha estado trabajando en la UNAD como tutor virtual en la Escuela de Ingeniería y en la Escuela de Educación. Se vinculó a la Fundación Universitaria del Área Andina - FUAA, sede Valledupar en el año 2015 y es profesor del programa de Ingeniería Civil.

ORCID: 0000-0002-2103-8636 